\title{
Laparoscopic Transvesical Mesh Removal and Vesicovaginal Fistula Repair
}

\author{
John R. Miklos, MD, Robert D. Moore, DO, Orawee Chinthakanan, MD, MPH \\ International Urogynecology Associates, Alpharetta, GA, USA (all authors).
}

\begin{abstract}
Background: Complications associated with anterior vaginal wall mesh used during transvaginal surgery for prolapse occur more frequently than in other areas of the vagina. A vesicovaginal fistula is one of the rare complications of anterior vaginal wall mesh surgery.
\end{abstract}

Case: A 69-year-old woman with a history of anterior vaginal wall prolapse developed a vesicovaginal fistula within days of undergoing anterior transvaginal mesh surgery. A transvesical laparoscopic approach was used to resect the bladder mesh. Then an extravesical vesicovaginal fistula repair was performed.

Conclusion: Laparoscopy can be an alternative to simultaneous bladder mesh removal and surgical correction of a vesicovaginal fistula.

Key Words: Vesicovaginal fistula, Mesh complications, Mesh erosion, Laparoscopic repair of vesicovaginal fistula, PROLIFT.

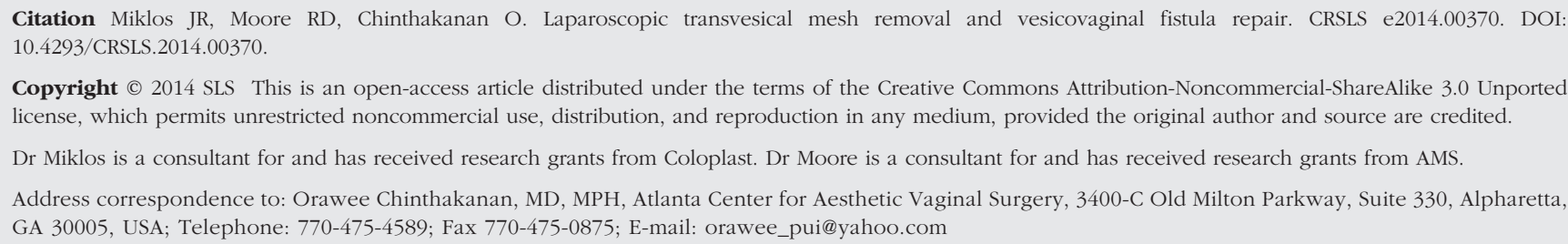

\section{INTRODUCTION}

Transvaginal mesh surgeries have become increasingly popular over the past few years in the treatment of vaginal prolapse. The more common complications associated with these meshes include vaginal pain, dyspareunia and mesh exposure. Urinary tract fistulas after transvaginal mesh surgeries have also been documented and have most commonly been addressed through vaginal and abdominal approaches. We report on the laparoscopic repair of a patient who was diagnosed with a vesicovaginal fistula (VVF) complicated with mesh, after undergoing anterior vaginal mesh surgery for the treatment of a cystocele.

\section{CASE STUDY}

The patient was a 69-year-old woman who presented to our office with a VVF for 5 weeks. The patient had an anterior PROLIFT (Gynecare, Newark, NJ) for anterior vaginal prolapse only 6 months after a failed anterior repair. The patient claimed that she started leaking urine copiously the day after surgery. A week later, she saw a urologist, who diagnosed her with a VVF. Further evaluation revealed mesh in the bladder, bladder wall, and vagina. The patient was referred to our practice for evaluation and management.

Evaluation in our office revealed vaginal mesh erosion in the bladder and extrusion in the vagina as well as a VVF that measured $2.0 \mathrm{~cm}$ (Figure 1). The patient was given an informed consent document and elected to proceed with a laparoscopic approach for mesh removal as well as VVF repair. The patient was taken to surgery 12 weeks after the initial placement of the anterior vaginal wall mesh.

Under general anesthesia, cystoscopy was performed, and a right ureteral stent was placed because of the close proximity of the fistula and the right ureter. Open laparoscopy was performed at the inferior edge of the umbilicus, where a $10-\mathrm{mm}$ port was placed to accommodate 

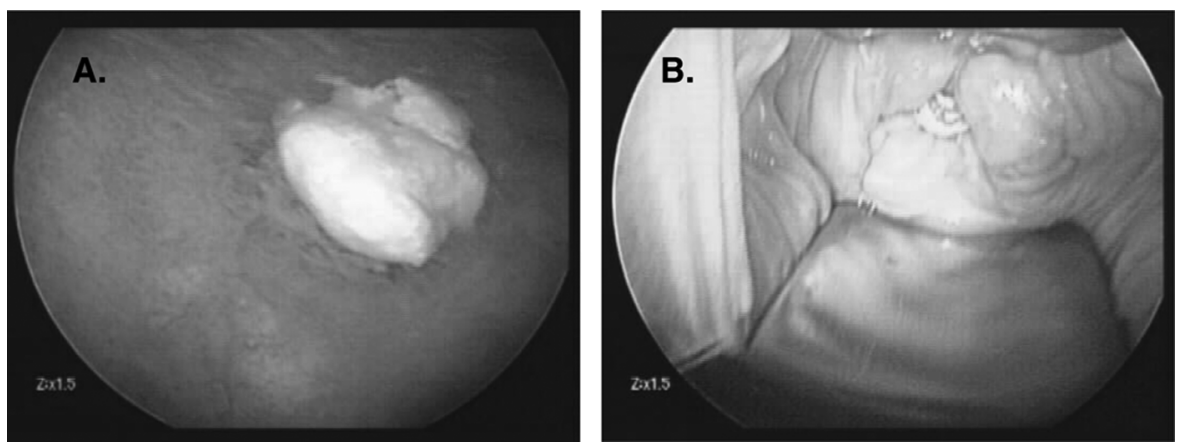

Figure 1. Mesh erosion in the bladder (A) and extrusion in the anterior vaginal wall (B) as well as a vesicovaginal fistula that measured $2 \mathrm{~cm}$.
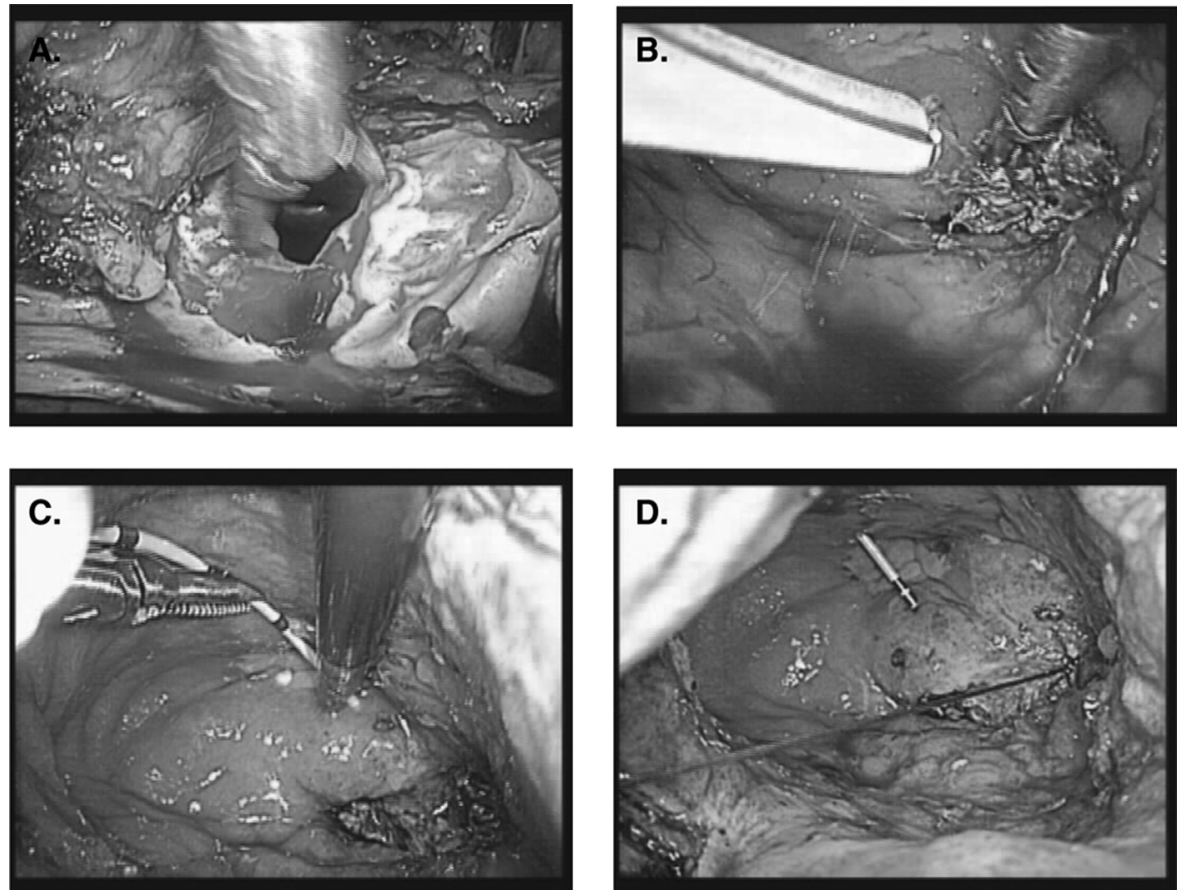

Figure 2. An intravesical approach of mesh removal and vesicovaginal fistula repair. (A) The bladder was incised vertically in the midline to allow intravesical access to synthetic mesh and fistula. (B) The mesh was then transected laparoscopically until the bladder mucosal edges were free of synthetic mesh, and the scarred edges of the bladder mucosa were excised. (C) The catheter was used to identify the right ureter. (D) The bladder mucosa was closed transversally with a single layer of delayed absorbable suture.

the laparoscope. Three other ports were placed under direct vision. A 5-mm port was placed suprapubically and in the right paramedian and a $10-\mathrm{mm}$ port in the left paramedian area. Once adhesions were lysed and adequate access to the vesicovaginal reflection was attained, an end-to-end anastomosis sizer was placed into the vagina to elevate the apex and to facilitate dissection. An intravesical approach was then taken by incising the bladder vertically in the midline to allow intravesical access to synthetic mesh and fistula (Figure 2A). The mesh was then transected laparoscopically until the bladder mucosal edges were free of synthetic mesh, and the scarred edges of bladder mucosa were excised (Figures 2B and 2C). A single-layer mucosa closure was performed with 3-0 delayed absorbable suture transversally (Figure 2D). At this time, the bladder dome was closed with a running 3-0 delayed absorbable suture in a double-layer fashion, and then the vesicovaginal dissection was performed to separate the bladder from the underlying vagina.

After complete separation of the vagina and the bladder, the vagina was closed with a single-layer closure using 
2-0 delayed absorbable sutures, and a second-layer closure of the bladder was performed extravesically using interrupted figure-of-eight sutures of 4-0 delayed absorbable sutures. At this point of the surgery, an ampule of 5 $\mathrm{mL}$ of indigo carmine was given intravenously. Cystoscopy and laparoscopy were performed simultaneously, and bladder integrity was confirmed after filling the bladder with $300 \mathrm{~mL}$ of normal saline solution. The ureteral stent was removed, and bilateral ureteral patency was confirmed. The bladder closure was also inspected from the vaginal side by direct inspection as well as using a white gauze sponge placed on the suture line to ensure no leakage of indigo carmine. After placement of the suprapubic catheter, the laparoscopic access ports were closed, and we directed our attention to the vagina. Excess mesh was trimmed from the vaginal epithelium, and closure of the vaginal epithelium was accomplished using 2-0 Vicryl (Ethicon Endo-Surgery, Blue Ash, OH) in an interrupted figure-of-eight fashion.

The patient returned 14 days after hospital discharge for cystoscopy. The bladder was retrograde filled, and the bladder and vaginal incisions were inspected. There was no evidence of bladder leakage, and the suprapubic catheter was removed. She followed up 6 months later without recurrence of the fistula.

\section{DISCUSSION}

Operations for mesh-related complications seem to occur most often after transvaginal placement in the anterior vaginal wall. ${ }^{1}$ Most commonly, reoperations are used to address vaginal mesh exposure problems and can be performed transvaginally. 2,3 However, more advanced problems, such as bladder mesh erosion and lower abdominal pain, have been addressed using abdominal or laparoscopic approaches. $4,5 \mathrm{VVF}$ repair with bladder mesh erosion, one of the most severe types of complications associated with the use of anterior vaginal wall mesh, have also been reported using both vaginal ${ }^{6}$ and abdominal ${ }^{7}$ approaches. Although laparoscopic VVF repairs have been reported previously, 8,9 we report the first case of simultaneous bladder mesh resection and VVF repair laparoscopically.

Although surgeons might recommend a vaginal approach, we chose a laparoscopic approach in this specific case for a number of reasons: (1) improved visualization of the mesh and meticulous dissection, allowing the comprehensive removal of mesh integrated into the bladder wall; (2) better visualization and thus a more meticulous closure of the bladder mucosa edges; and (3) our comfort using the laparoscopic approach while maintaining safety for the patient. The intravesical laparoscopic approach is a viable option, in experienced surgeons' hands, for bladder mesh removal.

\section{References:}

1. Nguyen JN, Jakus-Waldman SM, Walter AJ, White T, Menefee SA. Perioperative complications and reoperations after incontinence and prolapse surgeries using prosthetic implants. Obstet Gynecol. 2012;119(3):539-546.

2. Firoozi F, Goldman HB. Transvaginal excision of mesh erosion involving the bladder after mesh placement using a prolapse kit: a novel technique. Urology. 2010;75(1):203-206.

3. Khong S-Y, Lam A. Laparoscopic removal of mesh used in pelvic floor surgery. J Minim Invasive Gynecol. 2009;16(5):592594.

4. Pikaart DP, Miklos JR, Moore RD. Laparoscopic removal of pubovaginal polypropylene tension-free tape slings. JSLS. 2006; 10(2):220.

5. Maher C, Feiner B. Laparoscopic removal of intravesical mesh following pelvic organ prolapse mesh surgery. Int Urogynecol J. 2011;22(12):1593-1595.

6. Firoozi F, Ingber MS, Moore CK, Vasavada SP, Rackley RR, Goldman HB. Purely transvaginal/perineal management of complications from commercial prolapse kits using a new prostheses/grafts complication classification system. J Urol. 2012;187(5): $1674-1679$.

7. Yamada BS, Govier FE, Stefanovic KB, Kobashi KC. Vesicovaginal fistula and mesh erosion after Perigee (transobturator polypropylene mesh anterior repair). Urology. 2006; 68(5):1121.e5-1121.e7.

8. Nezhat CH, Nezhat F, Nezhat C, Rottenberg H. Laparoscopic repair of a vesicovaginal fistula: a case report. Obstet Gynecol. 1994;83(5):899-901.

9. Miklos J, Sobolewski C, Lucente V. Laparoscopic management of recurrent vesicovaginal fistula. Int Urogynecol J. 1999; 10(2):116-117. 